\title{
DISCUSSION
}

\section{A microstructurally based effective stress for unsaturated soils}

\author{
E. E. Alonso, J.-M. Pereira, J. VAUnAT and S. Olivella (2010). Géotechnique 60, No. 12, \\ $913-925, \mathrm{http}: / / \mathrm{dx} . \mathrm{doi} . \mathrm{org} / 10.1680 /$ geot.8.P.002
}

\section{E. J. Murray, Murray Rix Geotechnical, Stoke Golding, UK and V. Sivakumar, Queen's University Belfast, UK \\ The authors recognise the following important aspects of the 'controlling stress regime' in unsaturated soils, which are} worth re-emphasising.

(a) A dual stress regime exists in unsaturated soils and is commonly represented by the net stress $\left(\sigma-u_{\mathrm{a}}\right)$ and matric suction $\left(u_{\mathrm{a}}-u_{\mathrm{w}}\right)$.

(b) As an alternative to the net stress, an 'effective' or 'generalised' stress equation may be used but the suction is usually employed as a necessary additional component in the interpretation of experimental data and analysis.

(c) It is important to take account of the microstructure in unsaturated soils.

The authors utilise a Bishop-type control equation as the 'generalised' stress equation. They recognise that $\chi=S_{\mathrm{r}}$ is inappropriate for clayey soils, although this equality is widely quoted in the literature. They propose and examine the use of $\chi=S_{\mathrm{r}}^{\mathrm{e}}$ where $S_{\mathrm{r}}^{\mathrm{e}}$ is an effective degree of saturation determined from experimental data for both soil stiffness and shear strength for a variety of soils, and is developed from the concept of an equivalent pore pressure. The authors start their analysis from considerations of the behaviour of saturated soils that are controlled by Terzaghi's effective stress $\left(\sigma-u_{\mathrm{w}}\right)$ and attempt to extend the approach to the behaviour of unsaturated soils. An assumption is made that a Bishop-like stress equation may be used to replace Terzaghi's stress in analysis similar to that for saturated soils.

Implicit in the statement that the behaviour of unsaturated soils is controlled by a dual stress regime is the tendency of fine-grained soils, or soils with a high percentage of fines, to develop a bi-modal structure as the phases adjust and interact to minimise the thermodynamic potential. The structure may be idealised under equilibrium conditions as comprising aggregates of soil particles and water that are surrounded by air-filled voids. The intra-aggregate void spaces are small compared to the larger inter-aggregate spaces. However, there is no strict definition of the shape or size of the aggregates. The only requirement is that the soil particles and water are intrinsically linked. This is a thermodynamic necessity under equilibrium conditions. Inter-aggregate water in the larger pores will be drawn into the aggregates under the action of suction in order to achieve equilibrium and the suction in a soil will equalise throughout.

Mercury intrusion porosimetry (MIP) analysis on compacted kaolin has demonstrated that over a wide range of suctions, aggregates are saturated with water and the air is restricted to the larger inter-aggregate voids (Sivakumar et al., 2010). Monroy et al. (2010) also found this to be true for London clay. For a soil system in equilibrium, an applied external pressure (or stress regime) is balanced by the components of pressures and stresses associated with the phases and their interactions internal to the soil, thus both the deformation behaviour and the strength are dictated by the bi-modal structure and dual stress regime which this implies.
It is the overall net deformation and strength response to changing conditions of a soil in which practising engineers are likely to be most interested and not the intricacies of the influence of changes in the microstructure. From this point of view the development of a simple approach to predict such responses would be valuable. Unfortunately, in unsaturated fine-grained soils, the bi-modal structure results in different deformation and strength characteristics within and between the aggregates. Correlation of the parameter $S_{\mathrm{r}}^{\mathrm{e}}$ with overall deformations masks the important contributions of the components of the bi-modal structure. It is unclear how relevant this is for practical situations, but as examples of characteristics that are masked, the following phenomena have been observed in tests on fine-grained soils (e.g. Alonso et al., 1995; Thom et al., 2007; Murray \& Sivakumar, 2010; Sivakumar et al., 2010; Boyd \& Sivakumar, 2011).

(a) Wetting results in individual aggregates expanding into the inter-aggregate void spaces reducing the overall potential expansion of a specimen.

(b) Aggregates may take up water and expand during shearing, although the soil specimen may experience overall volume reduction.

(c) The aggregates act as deformable structures in which particle rearrangement takes place under changing conditions but the aggregate structure is not readily broken down.

(d) Shearing and deformations become concentrated within the inter-aggregate void spaces as a soil becomes drier.

(e) The deformation and strength behaviour of unsaturated soils is dependent on past stress history and test conditions and thus on an anisotropic stress regime dependent on the aggregate fabric.

It seems likely that the deformation and strength parameters obtained by examination of overall soil response to a 'generalised' stress equation, that does not adequately take account of a bi-modal structure, will not provide an accurate predictive tool. It would have to be shown that deviation from actual behaviour was within acceptable limits for practical purposes.

The bi-modal structure in fine-grained soils is synonymous with a dual stress regime comprising the net stress $\left(\sigma-u_{\mathrm{a}}\right)$ between the aggregates and Terzaghi's effective stress $\left(\sigma-u_{\mathrm{w}}\right)$ within the aggregates (Murray \& Sivakumar, 2010). The suction $\left(u_{\mathrm{a}}-u_{\mathrm{w}}\right)$ is the difference between the fluid pressures and controls the equalisation of air and water pressures. The 'effective' stresses $\left(\sigma-u_{\mathrm{a}}\right)$ and $\left(\sigma-u_{\mathrm{w}}\right)$ and suction $\left(u_{\mathrm{a}}-u_{\mathrm{w}}\right)$ constitute the three possible stress state variables and any two of the stress state variables may be used to describe the stress regime in unsaturated soils. Alternatively, one of the stress state variables, usually $\left(u_{\mathrm{a}}-u_{\mathrm{w}}\right)$ and some appropriate 'generalised' stress equation may be employed. However, it is important to recognise that the stress state variables act through different volumes. The conjugate stress and volumetric variables must be incorporated in a 'generalised' stress equation in order to satisfy 
thermodynamic principles. For instance, the net stress $\left(\sigma-u_{\mathrm{a}}\right)$ may be taken as acting through the total soil mass represented by the specific volume $v=V / V_{\mathrm{s}}$ along with the suction $s=\left(u_{\mathrm{a}}-u_{\mathrm{w}}\right)$ acting through the volume of the aggregates represented by the specific water volume $v_{\mathrm{w}}=\left(V_{\mathrm{w}}+V_{\mathrm{s}}\right) / V_{\mathrm{s}}$. Equation (23) represents this formulation

$$
\sigma_{\mathrm{c}}^{\prime}=\sigma-u_{\mathrm{g}}+s \frac{v_{\mathrm{w}}}{v}
$$

where, $\sigma_{\mathrm{c}}^{\prime}=$ the volumetric coupling stress

The equation may be derived by considering the minimisation of the thermodynamic potential at equilibrium, virtual work analysis or equilibrium analysis (Murray \& Sivakumar, 2010). The equation includes terms for all three phases including the solid phase. The use of $S_{\mathrm{r}}$ or $S_{\mathrm{r}}^{\mathrm{e}}$ does not comply with thermodynamic principles as the solid phase is not represented. It is the solid phase that gives a soil its strength and gives rise to the stresses. The volume of the solid phase must be included in any controlling stress equation. Equation (23) describes a dual stress regime based on the volumes through which the stresses act and should not be confused with the Bishop-like stress equation proposed by the authors, which represents a single 'effective' stress.

The authors justify the use of $S_{\mathrm{r}}^{\mathrm{e}} s$ in place of $S_{\mathrm{r}} s$ on the basis that the latter term becomes very large as $s$ increases and would compress the soil in an unrealistic way. This is true based on the assumption that both $S_{\mathrm{r}}^{\mathrm{e}} s$ and $S_{\mathrm{r}} s$ act through the total volume of soil. The term $s\left(v_{\mathrm{w}} / v\right)$ in equation (23) also becomes very large as $s$ increases, but it only acts through the aggregates comprising the volumes of soil particles and water which are of low volume compressibility, and does not act through the volume of the significantly more compressible air voids. Very large stresses due to suction are present in unsaturated soils at low degrees of saturation but these do not necessarily cause unrealistic compressive strains as they are restricted to the aggregates and not the whole soil mass. The large suction stresses explain the resilience of the aggregates and the preferential shearing between them as the suction increases. The aggregates act as large deformable particles.

Recent work (Murray \& Sivakumar, 2010) has demonstrated the following points.

(a) The strength of unsaturated soils may be examined and logically described using the volumetric coupling stress of equation (23). This allows apparent anomalies in experimental data to be explained.

(b) The load-deformation behaviour of unsaturated soils during shearing may be presented logically in dimensionless three-dimensional space.

(c) It is possible utilising the dual stress regime described by equation (23) to examine the anisotropic deformation behaviour within and between the aggregates in unsaturated soils.

(d) Strains at the micromechanical level in triaxial strength testing must be interpreted in terms of energy dispersion not merely displacement and shear straining. This requires thermodynamic concepts to be correctly applied.

It is re-emphasised that the behaviour of unsaturated soils is complex, and simplifying assumptions and analysis are probably a necessity for general usage by practising engineers who often wish only to determine the general magnitude of deformation or a range of likely strengths. In this respect the authors are to be commended. A deeper understanding of the intricacies of unsaturated soil behaviour should, however, lead to improvements in predictive tools and greater justification for simplified analysis.
The main points to arise from the discussion are listed below.

(a) Use of a single, all-encompassing 'effective' stress is not appropriate in unsaturated soils.

(b) Aggregation in fine-grained soils dictates the microstructure. Thus stress history as well as soil properties play an important role in soil behaviour.

(c) A detailed understanding of soil behaviour relies on analysis of a bi-modal structure in fine-grained soils, which is synonymous with a dual stress regime. Thermodynamics dictates that under equilibrium conditions the components of the dual stress regime act through specific volumes and the stresses cannot be taken in isolation from these conjugate volumes.

(d) In order to develop simplified analysis for practical purposes it is important to start from a sound knowledge base so that errors arising in simplification can be identified and kept to a minimum.

\section{Authors' reply}

The main objective of the paper was to propose an 'effective' stress framework for unsaturated soils which could overcome some significant difficulties observed in the application of a Bishop intergranular stress in which parameter $\chi$ is identified with the degree of saturation. The idea of defining an inter-granular or constitutive stress by adding a suction-based contribution to the net stress is retained as in Bishop's proposal.

The approach is intended to be useful in practice and therefore the purpose was to maintain a relationship between macroscopic states of stress and strain without entering into a more detailed analysis of the origin of deformations. Accordingly, the validation of the approach was made by interpreting macroscopic experimental data. It should be added that the study covers successfully a relatively large number of soils ranging from granular materials to highplasticity clays. It was also shown that the defined microstructural parameter was consistent with direct observations (mercury intrusion porosimetry) in the case of Boom clay compacted at two different dry densities.

A key point, however, is that the interpretation offered by the proposed constitutive stress is inspired by the observed structure of unsaturated (compacted) soils having some clay content. The rationale behind the proposal accepts simplifications in an effort to find a simple approach which, nevertheless, is intended to explain experimental results.

This explains that no specific consideration is given to isolating microstructural deformations and integrating them into an observable strain measure. However, the authors are aware of the need to examine in more detail the microstructure, especially when dealing with expansive clays or when attempting to predict 'non-mechanic' macro variables such as permeability and its evolution during wetting or drying. In fact, in one of the approaches used in these cases, two suction components (micro and macro) were distinguished in order to explore the effects of lack of internal equilibrium on observable strains (Alonso et al., 2011). Again, this approach may also be termed 'empirical', even if it maintains a physical support derived from an interpretation of microscopic features.

The discussers object that the proposal made is not backed by thermodynamics. This is not correct and it can be shown that the proposed constitutive stress is supported by an energetic approach. The authors did not intend to focus the paper on thermodynamics of unsaturated soils. Interested readers are referred to the work of Coussy et al. (2010), which discusses the thermodynamics of elastoplasticity for unsaturated soils. This work is highly complementary to the 
proposition made in the paper mentioned. In brief, Coussy et al. (2010) base their work on an energetic approach at the macroscopic scale and establish that the widely used Bishop's stress, the $\chi$ factor being equal to the degree of saturation, amounts to making strong assumptions on the deformation in both elastic and plastic regimes. This assumption can be called pore iso-deformation and consists in assuming that all pores deform identically, irrespective of their saturation state (and thus irrespective of the pressure to which they are subjected). This assumption is of course wrong. This is particularly the case for a soil presenting a double porosity structure, as emphasised by the discussers. Actually, it is widely observed that macropores (filled by air in many occasions) undergo the main part of the deformation whereas micropores (generally keeping their water-saturated state) remain approximately undeformed. An approach based on up-scaling methods and micromechanics (Chateau \& Dormieux, 2002; Dormieux et al., 2006) ends up in the same conclusion in the case of elastic behaviour. These two approaches (which adopt macroscopic and microscopic points of view) share the conclusion that the $\chi$ factor cannot be set equal to the degree of saturation of water unless strong assumptions are made.

Following the work of Coussy et al. (2010) it can be shown that in conceptual unsaturated soil media described by a solid skeleton of particles in contact through interfaces having their own energy, a gas phase and a liquid phase, the work input to the solid skeleton (in a triaxial case: $p$, average stress; $q$ : deviatoric stress) may be written, for an incompressible solid phase

$$
\begin{aligned}
\mathrm{d} w= & \left(p-u_{\mathrm{a}}\right) \mathrm{d} \varphi_{\mathrm{a}}+\left(p-u_{\mathrm{w}}\right) \mathrm{d} \varphi_{\mathrm{w}} \\
& +q \mathrm{~d} \varepsilon_{\mathrm{q}}-\phi_{0}\left(u_{\mathrm{a}}-u_{\mathrm{w}}\right) \mathrm{d} S_{\mathrm{r}}
\end{aligned}
$$

where $u_{\mathrm{a}}, u_{\mathrm{w}}, u_{\mathrm{a}}-u_{\mathrm{w}}$ are the air pressure, water pressure and suction respectively, $\phi_{0}$ is the initial porosity and $\mathrm{d} S_{\mathrm{r}}$ is the change in degree of saturation. The equation expresses that stresses $p-u_{\mathrm{a}}$ and $p-u_{\mathrm{w}}$ are work conjugate to the changes in air and water volumetric contents, $\varphi_{\mathrm{a}}$ and $\varphi_{\mathrm{w}}$, due to the sole deformation process.

In an elastoplastic scenario part of the work input, equation (24), is stored as elastic energy. The difference $(\mathrm{d} D)$ between total and elastic work is dissipated in heat. Consider now the dissipation energy in terms of plastic strain components. The plastic volumetric strain becomes $\mathrm{d} \varepsilon_{\mathrm{v}}^{\mathrm{p}}=$ $-\mathrm{d} \varphi_{\mathrm{w}}^{\mathrm{p}}-\mathrm{d} \varphi_{\mathrm{a}}^{\mathrm{p}}$, which assumes also plastic incompressibility of the solid phase. Moving forward towards an effective stress concept requires additional assumptions. The usual one consists in introducing a coefficient $\chi$ ranging from 0 to 1 such as

$$
\mathrm{d} \phi_{\mathrm{a}}^{\mathrm{p}}=-(1-\chi) \mathrm{d} \varepsilon_{\mathrm{v}}^{\mathrm{p}} ; \quad \mathrm{d} \phi_{\mathrm{w}}^{\mathrm{p}}=-\chi \mathrm{d} \varepsilon_{\mathrm{v}}^{\mathrm{p}}
$$

Setting $\chi$ equal to the degree of saturation is thus equivalent to assuming that pores filled by air deform equivalently to those filled by water. Without this assumption, dissipation writes

$$
\mathrm{d} D=p^{\mathrm{B}} \mathrm{d} \varepsilon_{\mathrm{v}}^{\mathrm{p}}+q \mathrm{~d} \varepsilon_{\mathrm{q}}^{\mathrm{p}}+\beta \mathrm{d} \alpha
$$

where $p^{\mathrm{B}}$ is Bishop's stress, defined by

$$
p^{\mathrm{B}}=p-(1-\chi) u_{\mathrm{a}}-\chi u_{\mathrm{w}}
$$

and $\alpha$ and $\beta$ refer to conjugate hardening variables. Parameter $\chi$ cannot be identified without further assumptions. It is reasonable to assume that $\chi$ is a function of the degree of saturation (but not solely) as stated by Bishop in his original paper. This explains why the authors used data on elastic and strength properties of unsaturated soils to obtain some evidence of this weighting function. In short, adopting a convenient form for $\chi$, interpreting experimental observations, is respectful of basic thermodynamics for unsaturated granular media.

Thermodynamics do not state that water and solid particles have to interact. On the contrary, the theoretical framework for continuum mechanics of porous materials has initially been settled assuming that there was no physicochemical interaction between the porous solid and its saturating fluid. If this occurs, it is clear that new stress measures have to be defined (e.g. see Vandamme et al. (2010) in the case of coal saturated by either methane or carbon dioxide, those gases presenting a sharp difference of adsorption properties on coal).

Additionally, it cannot be stated that the specific volume of solids has to enter into the definition of the constitutive stress. The reasoning leading to Bishop's stress presented above illustrates this point.

The authors agree with the final main four points which close the discussion.

A note on vocabulary: the term 'effective degree of saturation' introduced in the paper is questionable (its concept is, rather, a scaling parameter just as the $\chi$ function of Bishop's equation). In addition, $S_{\mathrm{r}}^{\mathrm{m}}$ is not an amount of water but a threshold value which describes microstructure. It may be more properly named 'microstructural parameter' It can be determined by techniques providing the distribution of pore sizes within the soil.

\section{REFERENCES}

Alonso, E. E., Lloret, A., Gens, A. \& Yang, D. Q. (1995). Experimental behaviour of highly expansive double-structure clay. Proc. 1st Int. Conf. on Unsaturated Soil, UNSAT95, Paris, France 1, 11-16.

Alonso, E. E., Romero, E. \& Hoffmann, C. (2011). Hydro-mechanical behaviour of compacted granular expansive mixtures. Experimental and constitutive study. Géotechnique 61, No. 4, 329-344, http://dx.doi.org/10.1680/geot.2011.61.4.329.

Boyd, J. L. \& Sivakumar, V. (2011). Experimental observations of the stress regime in unsaturated compacted clay when laterally confined. Géotechnique 61, No. 4, 345-363, http://dx.doi.org/ 10.1680/geot.2011.61.4.345.

Chateau, X. \& Dormieux, L. (2002). Micromechanics of saturated and unsaturated porous media, Int. J. Numer. Analyt. Methods Geomech. 26, No. 8, 831-844.

Coussy, O., Pereira, J. M. \& Vaunat, J. (2010). Revisiting the thermodynamics of hardening plasticity for unsaturated soils. Comput. Geotechnics 37, No. 1-2, 207-215.

Dormieux, L., Kondo, D. \& Ulm, F. J. (2006). Microporomechanics. Chichester, UK: Wiley.

Monroy, R., Zdravkovic, L. \& Ridley, A. (2010). Volumetric behaviour of compacted London Clay during wetting and loading. In Proc. 1st Eur. Conf. on Unsaturated Soils, E-UNSAT, Durham, UK. Unsaturated soils: advances in geo-engineering (eds D. G. Toll, C. Augarde, D. Gallipoli \& S. J. Wheeler), pp. 315-320. London, UK: Routledge.

Murray, E. J. \& Sivakumar, V. (2010). Unsaturated soils: a fundamental interpretation of soil behaviour. Oxford, UK. WileyBlackwell.

Sivakumar, V., Sivakumar, R., Murray, E. J., MacKinnon, P. \& Boyd, J. L. (2010). Mechanical behaviour of unsaturated kaolin with isotropic and anisotropic stress history. Part 1: wetting and compression behaviour. Géotechnique, 60, No. 8, 581-594, http://dx.doi.org/10.1680/geot.8.P.007.

Thom, R., Sivakumar, R., Sivakumar, V., Murray, E.J. \& MacKinnon, P. (2007). Pore size distribution of unsaturated compacted kaolin: the initial states and final states following saturation. Géotechnique 57, No. 5, 469-474, http://dx.doi.org/10.1680/ geot.2007.57.5.469.

Vandamme, M., Brochard, L., Lecampion, B. \& Coussy, O. (2010). Adsorption and strain: The $\mathrm{CO}_{2}$-induced swelling of coal. J. Mech. Phys. Solids 58, No. 10, 1489-1505. 